\title{
Intraoperative Neurophysiological Monitoring during Microvascular Decompression Surgery for Hemifacial Spasm
}

\author{
Sang-Ku Park, ${ }^{1 *}$ Byung-Euk Joo, ${ }^{2}$ Kwan Park $^{3}$ \\ Department of Neurology, Samsung Medical Center, Sungkyunkwan University School of Medicine, Seoul, Korea \\ Department of Neurology, ${ }^{2}$ Myongji Hospital, Hanyang University Medical Center, Goyang, Korea \\ Department of Neurosurgery, ${ }^{3}$ Samsung Medical Center, Sungkyunkwan University School of Medicine, Seoul, Korea
}

Hemifacial spasm (HFS) is due to the vascular compression of the facial nerve at its root exit zone (REZ). Microvascular decompression (MVD) of the facial nerve near the REZ is an effective treatment for HFS. In MVD for HFS, intraoperative neurophysiological monitoring (INM) has two purposes. The first purpose is to prevent injury to neural structures such as the vestibulocochlear nerve and facial nerve during MVD surgery, which is possible through INM of brainstem auditory evoked potential and facial nerve electromyography (EMG). The second purpose is the unique feature of MVD for HFS, which is to assess and optimize the effectiveness of the vascular decompression. The purpose is achieved mainly through monitoring of abnormal facial nerve EMG that is called as lateral spread response (LSR) and is also partially possible through Z-L response, facial F-wave, and facial motor evoked potentials. Based on the information regarding INM mentioned above, MVD for HFS can be considered as a more safe and effective treatment.

Key Words : Hemifacial spasm · Microvascular decompression surgery · Intraoperative neurophysiological monitoring.

\section{INTRODUCTION}

Hemifacial spasm (HFS) is defined as the tonic and clonic contraction of the muscles innervated by the ipsilateral facial nerve. Pathologically, HFS is caused by vascular compression of the facial nerve at its root exit zone from the brainstem. Therefore, microvascular decompression (MVD), which involves relieving neurovascular compression, is known as the curative treatment for $\mathrm{HFS}^{3,4)}$. MVD for HFS has been shown to be relatively safe, with a high cure rate between $70 \%$ and almost $100 \%{ }^{4,11,34)}$. In HFS, the abnormal muscle response can be elicited by stimulating one branch of the facial nerve and recording at the muscles innervated by the other facial nerve branch. This abnormal muscle response is the primary characteristic denoting HFS and is called the lateral spread response (LSR) $)^{36}$. The mechanisms underlying the LSR are unclear. Peripherally, compression of the facial nerve by a blood vessel causes injury of the myelin sheath, facilitating ectopic

- Received : November 27, 2018 •Revised : March 20, $2019 \bullet$ Accepted : April 4, 2019

- Address for reprints : Kwan Park

Department of Neurosurgery, Samsung Medical Center, Sungkyunkwan University School of Medicine, 81 Irwon-ro, Gangnam-gu, Seoul 06351, Korea

Tel : +82-2-3410-3499, Fax : +82-2-3410-0048, E-mail : kwanmd.park@samsung.com, ORCID : https://orcid.org/0000-0002-3263-6594

${ }^{*}$ These authors contributed equally to the manuscript as first author.

This is an Open Access article distributed under the terms of the Creative Commons Attribution Non-Commercial License (http://creativecommons.org/licenses/by-nc/4.0) which permits unrestricted non-commercial use, distribution, and reproduction in any medium, provided the original work is properly cited. 
excitation and ephaptic transmission between individual facial nerve branches ${ }^{17,28,45)}$ whereas centrally, the hyperexcitability of the facial motor nucleus (FMN), triggered by antidromically propagated discharges, induces HFS ${ }^{24,29,43)}$.

The primary purpose of intraoperative neurophysiological monitoring (INM) during MVD for HFS is to prevent intraoperative injury to neural structures. As the vestibulocochlear nerve (CN VIII) is directly adjacent to the facial nerve, the $\mathrm{CN}$ VIII can be damaged during decompression. Therefore, INM of the brainstem auditory evoked potential (BAEP) is mandatory during MVD for HFS to prevent to postoperative hearing loss. Unlike other surgeries, INM during MVD for HFS can additionally assess and optimize vascular decompression, which is primarily achieved through monitoring the LSR. As disappearance of the LSR during MVD is known to be associated with effective facial nerve decompression for HFS, monitoring the LSR can help identify the offending vessel and improve the accuracy of the decompression procedure. The BAEP and LSR are the most important measures assessed by INM during MVD for HFS. In addition, facial electromyography (EMG) such as the Z-L response and free-running EMG, and the facial F-wave and facial motor evoked potentials, contribute to the effectiveness of MVD for HFS.

Therefore, we present BAEP, LSR, and additional facial EMG findings, including facial F-wave and facial motor evoked potentials, from studies that used INM primarily during MVD for HFS.

\section{INM STUDIES}

\section{BAEP (introduction/method/warning criteria)}

The most frequent complications of MVD surgery for HFS is postoperative hearing loss from injury to the CN VIII. Injury could occur through traction during cerebellar retraction, ischemia due to vasospasm during manipulation of the compressive vessel loops, mechanical or thermal trauma during vessel and nerve dissection, or compression from the inserted Teflon pad ${ }^{14,22,23)}$. Postoperative hearing loss after MVD was reported to range from 7.7 to $20 \%$ without BAEP monitoring ${ }^{31)}$. However, after the introduction of INM of BAEPs, postoperative hearing loss decreased to $2 \%$ or less ${ }^{31,37)}$. Therefore, INM of BAEPs is now considered essential during MVD for HFS.

\section{Methodology}

BAEPs are most often elicited by broadband click stimuli, typically generated by passing 100-microsecond duration electrical square pulses to the transducer that generates the acoustic signals. The stimulus intensity should be set at a level to produce clear BAEPs but not cause ear damage. A click intensity of $100 \mathrm{~dB}$ sound pressure level (SPL) or 60-70 dB hearing level (HL) is commonly utilized. To mask crossover responses, white noise at $60 \mathrm{~dB}$ SPL or $30-35 \mathrm{~dB}$ HL is applied to the contralateral ear. Clicks can be of two polarities, either condensation or rarefaction, depending on the initial movement of the diaphragm of the transducer. Since electrical artifacts are often problematic during INM, alternating click polarity may be useful to minimize stimulus artifacts. Stimulus rates of 5 to $30 \mathrm{~Hz}$ have been reported in the literature. The American Clinical Neurophysiology Society (ACNS) recommends a stimulus rate of $5-12 \mathrm{~Hz} / \mathrm{sec}$ because faster rates may degrade the BAEP waveform. In addition, the ACNS suggests that 500-1000 repetitions are generally required to obtain an interpretable and reproducible $\mathrm{BAEP}^{1)}$. However, a stimulus rate of $10 \mathrm{~Hz}$ in an average of 1000 trials requires approximately 100 seconds to obtain BAEPs. This relative increase in duration may limit the prevention of postoperative hearing loss (Fig. 1). With the development of an INM machine with a high signal-to-noise ratio, the disintegration of the waveform amplitude that occurs with a higher stimulation rate is significantly improved; we have found no significant difference in the disintegration of the waveforms obtained at an $\sim 40 \mathrm{~Hz} / \mathrm{sec}$ stimulation rate and those obtained at a $10 \mathrm{~Hz} / \mathrm{sec}$ stimulation rate. Furthermore, it is possible to obtain a reliable waveform with fewer trials. Joo et al. ${ }^{15)}$ reported reliable waveforms at 43.9 $\mathrm{Hz} / \mathrm{sec}$ in an average of 400 trials. By obtaining a reliable BAEP in less than 10 seconds using a real-time protocol during MVD for HFS, it is possible to detect injury to the CN VIII more quickly during MVD surgery, and thus significantly reduce postoperative hearing loss $(4.02 \%$ vs. $0.39 \%, p=0.002)$ (Table 1).

\section{Warning criteria}

BAEP monitoring has been used for many years and is known to reduce the risk of postoperative hearing loss. Despite unequivocal evidence, there is no consensus on the warning criteria to predict postoperative hearing loss. Polo et al. ${ }^{31)}$ postulated that a wave $\mathrm{V}$ latency prolongation of $0.6 \mathrm{~ms}$ 
represented a significant BAEP change likely to be associated with HL. Grundy et al." ${ }^{7)}$ suggested that the surgeon should be
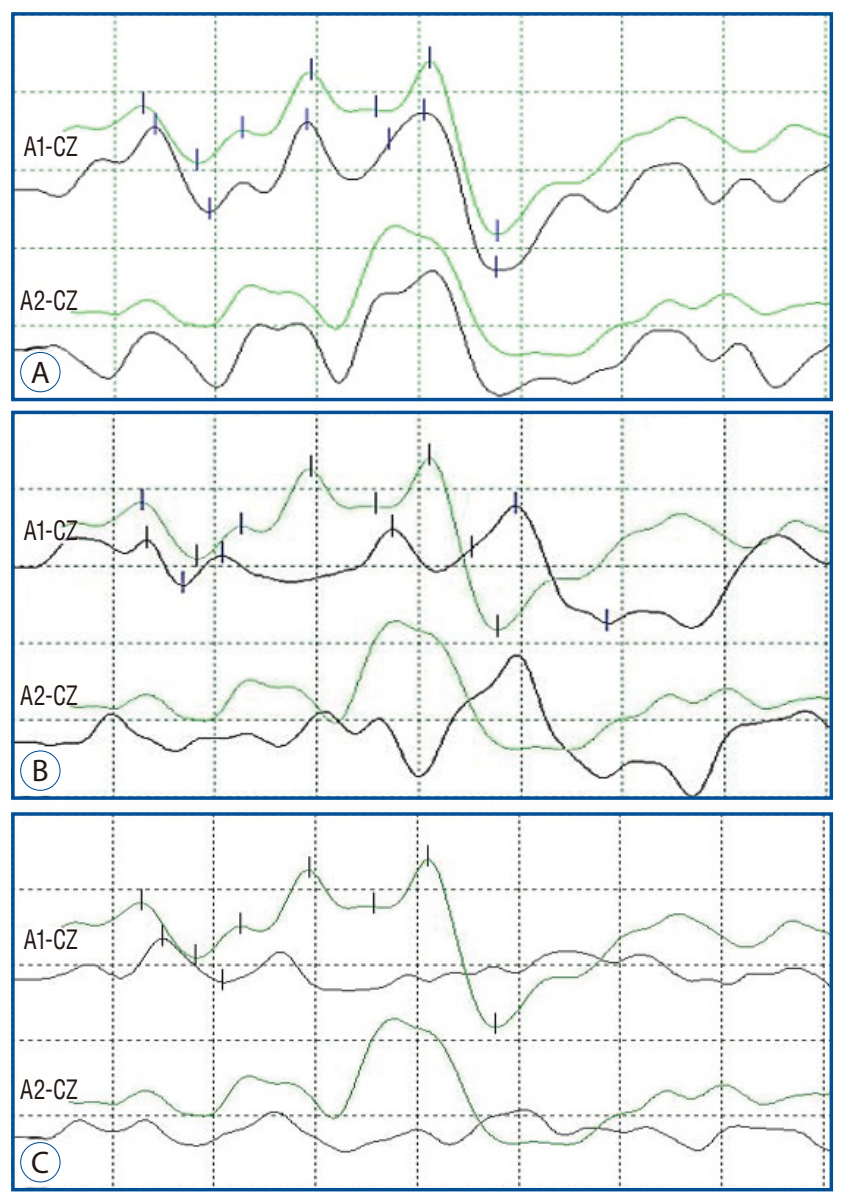

Fig. 1. Example of consecutive INM of the BAEP on left side using a stimulation rate of $10 \mathrm{~Hz} / \mathrm{sec}$ and 1000 averaging times (green line : baseline BAEP; black line : obtained BAEP). A : First BAEP showing minimal wave $V$ change. $B$ : Second BAEP showing a slight change in wave $V$ (the latency of wave $V$ was delayed by $1.36 \mathrm{~ms}$ with a minimal decrease in the amplitude. $C$ : Third BAEP showing a critical change in wave $V$ (loss of wave V). INM : intraoperative neurophysiological monitoring, BAEP : brainstem auditory evoked potential. warned when a $1.5-\mathrm{ms}$ delay in wave $\mathrm{V}$ is observed. In contrast, Hatayama and Møller ${ }^{9)}$ reported that prolongation of wave $\mathrm{V}$ latency was always accompanied by a reduction in wave $\mathrm{V}$ amplitude. Also, they presented that amplitude decrements of wave $\mathrm{V}$ were observed more significantly than latency prolongation of wave $\mathrm{V}$ in patients with postoperative hearing loss ${ }^{9)}$. Consequently, they suggested that a decrease in amplitude is a more reliable indicator for postoperative hearing loss. Recently, Thirumala et al. ${ }^{39)}$ reported that the loss of wave $\mathrm{V}$ during MVD was a significant warning criterion for predicting postoperative hearing loss. They noted that the probability of hearing loss was $60 \%$ with a persistent loss of wave $\mathrm{V}, 25 \%$ with a transient loss, and $10.2 \%$ when a significant change of wave $\mathrm{V}$ was observed. Despite considerable research about significant warning signs, some results are contradictory. Thus, many researchers still consider a latency prolongation of $1 \mathrm{~ms}$ or an amplitude decrement of at least $50 \%$ on two successive trials as the warning sign ${ }^{2}$. Because inaccurate warning criteria of BAEP can delay operation time and even lead to postoperative hearing loss with unnecessary additional manipulation, it is critical to establish the key warning signs during INM for predicting postoperative hearing loss. Park et al. ${ }^{30)}$ reported the critical warning criteria of BAEP for predicting postoperative hearing loss in 932 patients with HFS. They obtained the BAEP within approximately 9.1 seconds using a $43.9 \mathrm{~Hz} / \mathrm{sec}$ stimulation rate in an average of 400 trials. When applying the real-time BAEP protocol, postoperative hearing loss occurred in 11 patients (1.2\%), and was most commonly observed in patients with a persistent loss of wave V (6 of 11, 54.5\%). Furthermore, patients who showed a transient loss or a latency prolongation of $1 \mathrm{~ms}$ with an amplitude decrement of a least 50\% had postoperative hearing loss at a frequency of $7.4 \%$ and $2.0 \%$, respectively. Otherwise, of the 194 patients that experienced only latency prolongation

Table 1. Protocol comparison of INM of BAEPs and postoperative hearing loss

\begin{tabular}{|c|c|c|c|}
\hline INM BAEP & Previous protocol* & Real-time protocol ${ }^{\dagger}$ & $p$-value \\
\hline Stimulation rate & $26.9 \mathrm{~Hz}$ & $43.9 \mathrm{~Hz}$ & \\
\hline Average number of trials & $1000-2000$ & 400 & \\
\hline Time to obtain BAEP & About 37.1-74.3 seconds & About 9.1 seconds & \\
\hline Warning criteria of wave $V$ & 1 ms latency prolongation or a 50\% decrease in amplitude & $50 \%$ decrease in amplitude & \\
\hline Postoperative hearing loss & $4.02 \%$ & $0.39 \%$ & 0.002 \\
\hline
\end{tabular}

*Protocol used in our previous study ${ }^{14)}$. ${ }^{\dagger}$ Protocol used in our recent study ${ }^{15)}$. INM : intraoperative neurophysiological monitoring, BAEP : brainstem auditory evoked potential 
(pl ms) without amplitude reduction greater than 50\% during MVD surgery-including 30 patients who showed latency prolongation $>2 \mathrm{~ms}$ - no postoperative hearing loss was observed (Fig. 2). This study also provided validity data of the warning criteria of BAEP for predicting postoperative hearing loss during MVD surgery for patients with HFS. They reported that the permanent loss of wave $V$ showed the highest specificity of $99.4 \%$ for predicting postoperative hearing loss; transient loss and latency prolongation (1 ms) with amplitude reduction (50\%) also showed high accuracy (Table 2). In summary, the authors concluded that the currently adopted 'significant warning signs,' such as a latency prolongation of more than $1 \mathrm{~ms}$ or an amplitude decrement of at least more than $50 \%$, are inappropriate for preventing postoperative hearing loss during MVD surgery. Moreover, since a single warning sign would be inaccurate for preventing postoperative hearing loss during MVD surgery, they suggested a 'sliding scale' for the critical warning signs of BAEP as follows : 1) the observation sign (attention sign) : a latency prolongation of $1 \mathrm{~ms}$ without an amplitude decrement of at least 50\%, 2) the warning sign: a latency prolongation of $1 \mathrm{~ms}$ with an amplitude decrement of at least 50\%, and 3) the critical sign : a loss of wave V. When the observation sign is observed during MVD surgery, the neurophysiologist notifies the surgeon immediately, but no corrective maneuvers are performed. The surgeon is again notified when the warning or critical sign appears, and institute more aggressive measures to prevent injury of the $\mathrm{CN}$ VIII when the critical sign occurs.



\begin{tabular}{|c|c|c|c|c|c|c|c|}
\hline Set & I & I-I' & I-III & III-V & $\mathrm{I}-\mathrm{V}$ & $V^{\prime}-V^{\prime}$ & V \\
\hline t 21 & $2.40 \mathrm{~ms}$ & 0.16 킮 & $3 \mathrm{~ms}$ & $1.93 \mathrm{~ms}$ & $5.07 \mathrm{~ms}$ & 0.36 킴 & $7.47 \mathrm{~m}$ \\
\hline 20 & $3 \mathrm{~ms}$ & 0.20 킮 & $7 \mathrm{~ms}$ & $2.00 \mathrm{~ms}$ & $5.07 \mathrm{~ms}$ & 0.36 킴 & $7.50 \mathrm{~m}$ \\
\hline t 19 & $3 \mathrm{~ms}$ & 6 킮 & $\mathrm{ms}$ & $3 \mathrm{~ms}$ & $3 \mathrm{~ms}$ & 0.40 킮 & $7.37 r$ \\
\hline 18 & $2.23 \mathrm{~ms}$ & 13 킮 & $00 \mathrm{~ms}$ & $2.17 \mathrm{~ms}$ & $5.17 \mathrm{~ms}$ & 0.39 킴 & 40 \\
\hline 17 & $2.27 \mathrm{~ms}$ & 0.11 킮 & $3 \mathrm{~ms}$ & $2.00 \mathrm{~ms}$ & $5.03 \mathrm{~ms}$ & 0.42 킮 & $7.30 r$ \\
\hline$t 16$ & $\mathrm{~ms}$ & 3 킴 & $3 \mathrm{~ms}$ & $7 \mathrm{~ms}$ & $0 \mathrm{~ms}$ & 0.40 킴 & 7. \\
\hline et 15 & $2.27 \mathrm{~ms}$ & 0.19 킮 & $3 \mathrm{~ms}$ & $1.80 \mathrm{~ms}$ & $5.03 \mathrm{~ms}$ & 0.36 킴 & 7.30 \\
\hline Set 14 & $2.50 \mathrm{~ms}$ & 0.13 킮 & $3.23 \mathrm{~ms}$ & $1.90 \mathrm{~ms}$ & $5.13 \mathrm{~ms}$ & 0.35 킮 & $7.63 n$ \\
\hline 13 & $\mathrm{~ms}$ & 2 킴 & $\mathrm{ms}$ & $\mathrm{ms}$ & $3 \mathrm{~ms}$ & 0.28 킮 & 8 \\
\hline Se & ns & 9 킴 & $\mathrm{ns}$ & ns & $\mathrm{ns}$ & 3 킴 & $8.23 \mathrm{~m}$ \\
\hline Set 8 & $2.73 \mathrm{~ms}$ & 0.05 킮 & $3.07 \mathrm{~ms}$ & $2.50 \mathrm{~ms}$ & $5.57 \mathrm{~ms}$ & 0.22 킴 & $8.30 n$ \\
\hline Set 7 & $2.27 \mathrm{~ms}$ & 0.10 킮 & $3.67 \mathrm{~ms}$ & $1.97 \mathrm{~ms}$ & $5.63 \mathrm{~ms}$ & 0.24 킮 & $7.90 n$ \\
\hline Set 6 & 15 & 3 킴 & $3 \mathrm{~ms}$ & ns & ns & 0.35 킴 & $7.73 \mathrm{~m}$ \\
\hline Set 5 & $2.33 \mathrm{~ms}$ & 0.14 킮 & $3.03 \mathrm{~ms}$ & $2.13 \mathrm{~ms}$ & $5.17 \mathrm{~ms}$ & 0.38 킴 & $7.50 \mathrm{r}$ \\
\hline Set 4 & $2.23 \mathrm{~ms}$ & 0.20 킮 & $2.93 \mathrm{~ms}$ & $2.07 \mathrm{~ms}$ & $5.00 \mathrm{~ms}$ & 0.38 킮 & $7.23 n$ \\
\hline Set & $2.27 \mathrm{~ms}$ & 0.16 킮 & $7 \mathrm{~ms}$ & $1.97 \mathrm{~ms}$ & $\mathrm{~ms}$ & 0.47 킮 & 6 \\
\hline Set2 & $2.27 \mathrm{~ms}$ & 0.19 킮 & $2.50 \mathrm{~ms}$ & $\mathrm{~ms}$ & $\mathrm{~ms}$ & 0.48 킮 & $6.80 \mathrm{~ms}$ \\
\hline Set 1 & $1.93 \mathrm{~ms}$ & 0.14 킮 & $2.50 \mathrm{~ms}$ & $1.87 \mathrm{~ms}$ & $4.37 \mathrm{~ms}$ & 0.45 킮 & 6.30 \\
\hline
\end{tabular}
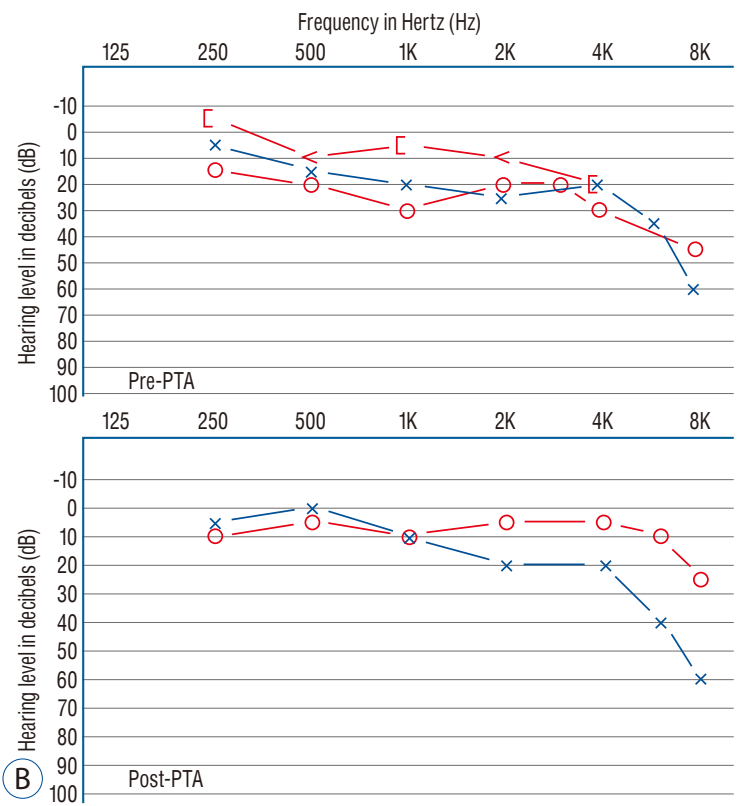

Fig. 2. A representative case showing only latency prolongation $(\geq 1 \mathrm{~ms}$ ) without a significant change in amplitude (the latency of wave $V$ was delayed by $2.00 \mathrm{~ms}$ from $6.30 \mathrm{~ms}$ to $8.30 \mathrm{~ms}$ with a minimal decrease in the amplitude). The patient in this example did not experience postoperative hearing loss (the average pre-PTA threshold was $22.5 \mathrm{~dB}$, and the average post-PTA threshold was $6.25 \mathrm{~dB}$ ). A : The INM of BAEP during MVD surgery (blue arrow, blue box : baseline of wave $V$; red arrow, red box : wave $V$ showing maximal prolongation in latency). $B$ : Pure tone audiometry of the patients obtained prior to surgery and 7 days postoperatively. PTA : pure tone audiometry, INM : intraoperative neurophysiological monitoring, BAEP : brainstem auditory evoked potential, MVD : microvascular decompression.

Table 2. Validity of the warning criteria of BAEP to predict postoperative hearing loss

\begin{tabular}{|c|c|c|c|}
\hline Warning criteria of the wave $\mathrm{V}$ & Latency prolongation ( $\geq 1 \mathrm{~ms}$ ) with amplitude decrement ( $\geq 50 \%)$ & Transient loss & Permanent loss \\
\hline Sensitivity & 0.909 & 0.727 & 0.545 \\
\hline Specificity & 0.865 & 0.967 & 0.994 \\
\hline Positive predictable value & 0.075 & 0.211 & 0.545 \\
\hline Negative predictable value & 0.999 & 0.996 & 0.995 \\
\hline
\end{tabular}

These results are from Park et al. ${ }^{30}$. BAEP : brainstem auditory evoked potential 


\section{Facial nerve EMG}

\section{LSR}

The LSR is elicited by stimulation of the facial nerve branch and is recorded from facial muscles innervated by another branch. Numerous studies have demonstrated a positive correlation between the intraoperative disappearance of LSR and favorable outcome in patients undergoing MVD for HFS; therefore, LSR has been used as an indicator of complete facial nerve decompression ${ }^{8,13,26)}$. The LSR usually disappears after microvascular decompression in patients with HFS, with the nerve considered to be adequately decompressed ${ }^{10,12,19)}$. However, controversial findings, such as LSR absence before MVD or LSR persistence after MVD, have been reported ${ }^{18,40-42)}$. Furthermore, several studies suggested that residual LSR after MVD was not related to long-term outcome of HFS; conversely, other studies concluded that repeated MVD was necessary because residual LSR indicates insufficient decompression ${ }^{40,41,44)}$. Therefore, the practical value of LSR disappearance as an indicator of adequate decompression remains controversial.

\section{Methodology}

Generally, the temporal or zygomatic branch of the facial nerve, approximately $3 \mathrm{~cm}$ lateral to the lateral margin of the orbit, is stimulated for recording the LSR. The stimulating needle electrodes are inserted intradermally over the temporal or zygomatic branches of the facial nerve, and the direction of stimulation with paired needles is centripetal towards the brainstem, with the cathode positioned proximally ${ }^{25,26,43)}$. A 0.3 -ms pulse wave with an intensity of 5 to $25 \mathrm{~mA}$ is used. The facial nerve EMG is recorded from the frontalis, orbicularis oculi, orbicularis oris, and the mentalis muscle. Although the LSR has been considered a reliable diagnostic tool and an indicator of complete nerve decompression in HFS, the question of whether INM of the LSR is a reliable indicator for complete facial nerve decompression has been arisen because of controversial findings such as LSR absence before MVD and LSR persistence after MVD. Recently, Lee et al. ${ }^{21)}$ suggested that a new LSR monitoring method shows greater reliability. To improve reliability, they conducted preoperative LSR monitoring at the outpatient clinic to map the facial nerve branch. After identifying the patient-specific location, they stimulated the facial nerve branch in the centrifugal direction with the anode located proximally over the area just anterior to the mandibular fossa and the cathode located distally in the temporal or zygomatic branch of the facial nerve (Fig. 3). A comparison of

(B)

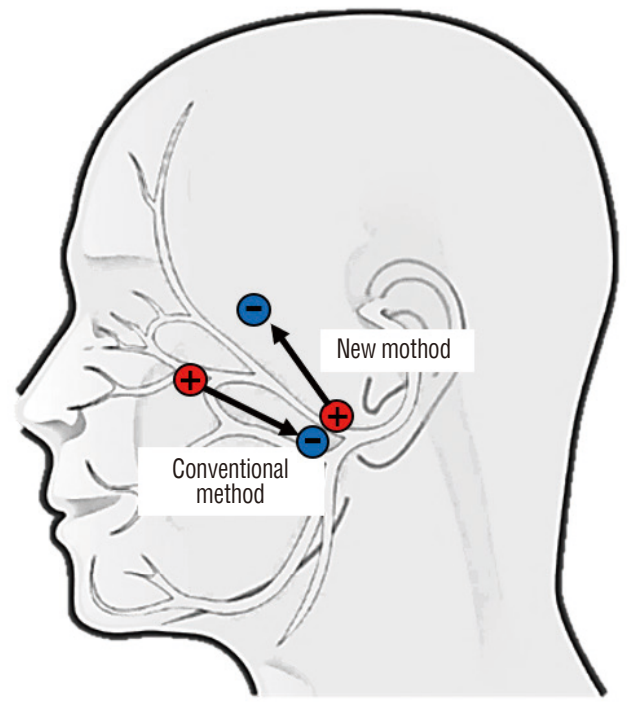

Fig. 3. A : Facial nerve mapping performed preoperatively. The locations of maximal LSR elicitation were divided into three regions. B : The direction of stimulation in the conventional and the new methods. In the conventional method, electrodes are placed in the temporal or zygomatic branch of the facial nerve, about $3 \mathrm{~cm}$ lateral to the lateral margin of the orbit, and centripetal impulses are transmitted towards the brainstem with the cathode positioned proximally. In contrast, electrodes were inserted intradermally with the anode located proximally over the area just anterior to the mandibular fossa and the cathode located distally in the temporal branch of the facial nerve in the new method. The direction of stimulation was centrifugal outwards from the brainstem. $\mathrm{F}$ : the direction towards the frontalis muscle, which was almost vertical with respect to the anode, $\mathrm{O}$ : the direction towards the orbicularis oculi muscle, F-O : in between F and O, LSR : lateral spread response. 
Table 3. Comparison of efficacy of LSR monitoring by using the conventional and new methods

\begin{tabular}{lccc}
\hline & $\begin{array}{c}\text { Conventional } \\
\text { method }\end{array}$ & New method & p-value \\
\hline Disappearance of LSR & $61.8 \%$ & $98.2 \%$ & 0.0012 \\
Persistence of LSR & $29.1 \%$ & $1.8 \%$ & 0.0051 \\
No LSR & $9.1 \%$ & $0.0 \%$ & $<0.0001$ \\
\hline
\end{tabular}

These results are from Lee et al. ${ }^{211}$. LSR : lateral spread response

the conventional and new method of LSR monitoring showed that the new method showed a significantly higher LSR disappearance after MVD (98.2\% vs. $61.8 \%, p=0.0012)$. Furthermore, the persistence of the LSR after MVD and the absence of the LSR was significantly lower with the new method (1.8\% vs. $29.1 \%, p=0.0051 ; 0.0 \%$ vs. $9.1 \%, p<0.0001$, respectively) (Table 3).

\section{Practical value}

INM of the LSR is known to be useful for identifying the offending vessels and for confirming whether the facial nerve has been decompressed ${ }^{26,27)}$. Some authors concluded that the reduction of the LSR amplitude might be not associated with bad outcomes even if the LSR did not completely disappear ${ }^{355}$. On the other hand, others suggested that the prognostic value of LSR monitoring for predicting the long-term outcomes of HFS was still questionable. Some researchers have reported that the disappearance of the LSR after MVD does not always correlated with postoperative prognosis in HFS. In 40 patients who showed LSR disappearance intraoperatively after MVD, five had mild HFS at discharge and four had mild HFS at the 6 month follow-up ${ }^{16)}$. Thirumala et al. ${ }^{40)}$ reported that there was no difference in HFS between patients showing disappearance of the LSR and persistence of the LSR at long-term follow-up, although disappearance of the LSR was correlated with immediate spasm relief. This residual LSR could support the central mechanism of HFS pathophysiology, including the hyperexcitability of the FMN in the brainstem ${ }^{5,24,25)}$. In these studies, the pulsatile impulse to the REZ was immediately removed once the MVD was performed, and the FMN began to decline and normalize slowly over a few months and even a few years in some patients. Therefore, monitoring the LSR during MVD can confirm the offending vessel and guide adequate decompression in patients with HFS. However, constant communication between the neurophysiologist and neurosur- geon on interpreting LSR findings is important to achieve adequate decompression because some patients can show a residual LSR after adequate decompression, which may indicate positive long-term outcomes.

\section{Z-L response}

The Z-L response (ZLR) is an alternative intraoperative facial nerve EMG measurement for $\mathrm{HFS}^{47}$. It is evoked while the wall of the offending artery is electrically stimulated ${ }^{46,47)}$. As mentioned above, a residual LSR exists despite adequate decompression, and some patients occasionally show disappearance of the LSR before the REZ of the facial nerve during the dura opening and dissection of the arachnoid membrane is addressed. The ZLR is known to be useful when the LSR is unavailable or unstable. Unlike the LSR, the ZLR is an orthodromic facial nerve EMG potential. The ZLR is elicited when an intracranial electrical stimulation is applied to the offending artery located at the REZ. Since the ZLR disappears instantly after decompression of the offending artery in HFS, the ZLR has been known to be useful when LSR is absent before MVD or when a residual LSR is observed. In addition, the ZLR can help determine the offending vessel when multiple offending vessels exit ${ }^{46)}$.

\section{Methodology}

The subdermal needle reference electrodes are inserted into the frontal muscle, and the needle recording electrodes inserted into the orbicularis oculi, orbicularis oris, and mentalis muscles. The stimulating electrode is a non-invasive concentric electrode, which was used intracranially. It can be considered the bipolar mode. Before detaching the offending artery from the facial nerve, the stimulating electrode is placed on the offending artery wall within $5 \mathrm{~mm}$ from the compression site of the REZ, a square impulse $(1-2 \mathrm{~mA}, 0.2 \mathrm{~ms}, 3 \mathrm{~Hz})$ is delivered, and facial EMG recorded. This recording procedure is repeated for every offending vessel until the facial nerve is completely decompressed.

\section{Practical value}

The ZLR may be useful when the LSR is unavailable or unstable. Zheng et al. ${ }^{47)}$ suggested that the combination of the LSR and ZLR provides more useful information than does the LSR alone because the ZLR may be the only useful INM facial EMG measurement in some cases. In addition, the ZLR can 
define the offending vessel when multiple offending vessels are present. Among the patterns of neurovascular compression, the ZLR was shown to be particularly useful for finding the offending vessel, which was concealed by the vertebral artery of the tandem type ${ }^{46}$. However, the ZLR should be used with careful consideration. As mentioned above, the ZLR is an orthodromic facial EMG measurement elicited form current spread to facial nerve form electrically stimulated arterial wall that contacts the facial nerve within several millimeters. Therefore, the ZLR would be elicited irrespective of the location of stimulation along the course of the artery if there was any mechanical contact between electrically stimulated arteries and the facial nerve ${ }^{38)}$. For example, if the distal cisternal portion of the offending arteries contacted the distal facial nerve in addition to vascular contact at the REZ, the ZLR can persist despite the disappearance of the LSR after complete decompression.

\section{Free-running EMG (Fr EMG)}

Fr EMG provides information regarding mechanical or thermal facial nerve injury. Nerve injury is manifested as sustained, high-frequency neurotonic discharges in facial nerve EMG. If this activity is detected, the neurophysiologist can provide immediate feedback to the neurosurgeon to allow operative maneuver changes to avoid nerve injury. Fr EMG activity in muscles innervated by the facial nerve was mainly studied in cerebellopontine angle surgery. Romstöck et al. ${ }^{33)}$ proposed a classification system for the patterns of facial nerve Fr EMG ${ }^{31}$ by separating spikes, bursts, and three different kinds of train-patterns with respect to waveform and frequency characteristics. The term "train" was introduced for sustained periodic EMG activity that lasts for seconds. Three typical train patterns with specific rhythmic features were observed. The A-train is the most important of these patterns. This train is a distinct EMG waveform of sinusoidal pattern that has maximum amplitudes ranging from 100 to $200 \mu \mathrm{V}$, and a frequency up to $210 \mathrm{~Hz}$. Their duration varies between milliseconds and several seconds. They can display as a single long pattern, or as a salvo of short A-trains. The occurrence of A-trains was shown to be associated with postoperative facial palsy with high sensitivity (86\%) and specificity (89\%) during cerebellopontine angle tumor surgeries ${ }^{32,33)}$. In patients with HFS, irregular Fr EMG activity can be spontaneously elicited during MVD, especially when saline is injected onto the facial nerve. When the ratio of post-MVD to pre-MVD Fr EMG activity was calculated to assess Fr EMG activity, Fr EMG activity ratios of $\geq 50 \%$ were reported to be associated with a greater likelihood of a residual LSR in patients with HFS').

\section{Facial F-wave}

The facial F-wave represents the backfiring of the facial motor neurons after being activated antidromically. F-wave activity was shown to be an index of motor neuron excitability ${ }^{20)}$. F-wave persistence appearance was reported in patients with HFS $^{12)}$ and found to decrease after adequate MVD, albeit with delay as long as 2 years. It is possible to record changes in elicitability of the facial F-wave during MVD. Immediate changes in hyperexcitability of the FMN can be observed by monitoring changes in F-wave elicitability. Facial F-waves can be obtained from the mentalis muscle by stimulating the mandibular branch of the facial nerve.

\section{Facial motor evoked potentials (facial MEP)}

Although uncommonly performed, myogenic facial MEP elicited through transcranial electric stimulation can be used to monitor the functional integrity of the FMN during MVD surger $y^{5}$. Facial MEP monitoring in patients with HFS has remarkable characteristics due to hyperexcitability of the FMN. Also, the stimulation threshold for eliciting a facial MEP response has been observed to increase after successful decompression of the facial nerve. Therefore, the facial MEP threshold before and after MVD can be used as an indicator of a successful MVD.

\section{CONCLUSION}

Postoperative HL due to injury of CN VIII is a common complication during MVD for HFS. This complication can be greatly reduced with the advent of INM of the BAEP. Unlike other surgeries, INM during MVD for HFS has a unique purpose; namely, to assess and optimize the vascular decompression for HFS. This is achieved through monitoring the LSR, ZLR, facial F-wave, and facial MEP. Increased knowledge and understanding of INM in MVD for HFS will improve safety and provide more effective treatment. 


\section{CONFLICTS OF INTEREST}

No potential conflict of interest relevant to this article was reported.

\section{INFORMED CONSENT}

This type of study does not require informed consent.

\section{AUTHOR CONTRIBUTIONS}

\author{
Conceptualization : SKP, BEJ \\ Data curation : SKP, BEJ, KP \\ Formal analysis : SKP, BEJ \\ Funding acquisition : KP \\ Methodology : SKP, BEJ \\ Project administration : KP \\ Visualization : BEJ \\ Writing - original draft : SKP, BEJ \\ Writing - review \& editing : SKP, BEJ, KP
}

\section{References}

1. American Clinical Neurophysiology Society : Guideline 9C: guidelines on short-latency auditory evoked potentials. J Clin Neurophysiol 23 : 157-167, 2006

2. American Electroencephalographic Society : Guideline eleven: guidelines for intraoperative monitoring of sensory evoked potentials. J Clin Neurophysiol $11:$ 77-87, 1994

3. Barker FG 2nd, Jannetta PJ, Bissonette DJ, Shields PT, Larkins MV, Jho HD : Microvascular decompression for hemifacial spasm. J Neurosurg 82 : 201-210, 1995

4. Chung SS, Chang JW, Kim SH, Chang JH, Park YG, Kim DI : Microvascular decompression of the facial nerve for the treatment of hemifacial spasm: preoperative magnetic resonance imaging related to clinical outcomes. Acta Neurochir (Wien) 142 : 901-906; discussion 907, 2000

5. Fernández-Conejero I, Ulkatan S, Sen C, Deletis V : Intra-operative neurophysiology during microvascular decompression for hemifacial spasm. Clin Neurophysiol 123 : 78-83, 2012

6. Fukuda M, Takao T, Hiraishi T, Fujii Y : Free-running EMG monitoring during microvascular decompression for hemifacial spasm. Acta Neurochir (Wien) 157 : 1505-1512, 2015

7. Grundy BL, Jannetta PJ, Procopio PT, Lina A, Boston JR, Doyle E : Intraoperative monitoring of brain-stem auditory evoked potentials. J Neu- rosurg $57: 674-681,1982$

8. Haines SJ, Torres $\mathrm{F}$ : Intraoperative monitoring of the facial nerve during decompressive surgery for hemifacial spasm. J Neurosurg 74 : 254257, 1991

9. Hatayama T, Møller AR : Correlation between latency and amplitude of peak $V$ in the brainstem auditory evoked potentials: intraoperative recordings in microvascular decompression operations. Acta Neurochir (Wien) $140: 681-687,1998$

10. Hatem J, Sindou M, Vial C : Intraoperative monitoring of facial EMG responses during microvascular decompression for hemifacial spasm. Prognostic value for long-term outcome: a study in a 33-patient series. Br J Neurosurg 15 : 496-499, 2001

11. Hyun SJ, Kong DS, Park K : Microvascular decompression for treating hemifacial spasm: lesion learned from a prospective study of 1,174 operations. Neurosurg Rev 33 : 325-334, 2010

12. Ishikawa M, Ohira T, Namiki J, Kobayashi M, Takase M, Kawase T, et al. : Electrophysiological investigation of hemifacial spasm after microvascular decompression: $F$ waves of the facial muscles, blink reflexes, and abnormal muscle responses. J Neurosurg 86 : 654-661, 1997

13. Isu T, Kamada K, Mabuchi S, Kitaoka A, Ito T, Koiwa M, et al. : Intraoperative monitoring by facial electromyographic responses during microvascular decompressive surgery for hemifacial spasm. Acta Neurochir (Wien) 138 : 19-23; discussion 23, 1996

14. Jo KW, Kim JW, Kong DS, Hong SH, Park K : The patterns and risk factors of hearing loss following microvascular decompression for hemifacial spasm. Acta Neurochir (Wien) 153 : 1023-1030, 2011

15. Joo BE, Park SK, Cho KR, Kong DS, Seo DW, Park K : Real-time intraoperative monitoring of brainstem auditory evoked potentials during microvascular decompression for hemifacial spasm. J Neurosurg 125 : 1061-1067, 2016

16. Joo WI, Lee KJ, Park HK, Chough CK, Rha HK : Prognostic value of intraoperative lateral spread response monitoring during microvascular decompression in patients with hemifacial spasm. J Clin Neurosci 15 : 1335-1339, 2008

17. Kameyama S, Masuda H, Shirozu H, Ito Y, Sonoda M, Kimura J : Ephaptic transmission is the origin of the abnormal muscle response seen in hemifacial spasm. Clin Neurophysiol 127 : 2240-2245, 2016

18. Kim CH, Kong DS, Lee JA, Park K : The potential value of the disappearance of the lateral spread response during microvascular decompression for predicting the clinical outcome of hemifacial spams: a prospective study. Neurosurgery $67: 1581-1588,2010$

19. Kim HR, Rhee DJ, Kong DS, Park K : Prognostic factors of hemifacial spasm after microvascular decompression. J Korean Neurosurg Soc 45 : 336-340, 2009

20. Kimura J : Current understanding of F-wave physiology in the clinical domain. Suppl Clin Neurophysiol 59 : 299-303, 2006

21. Lee S, Park SK, Lee JA, Joo BE, Kong DS, Seo DW, et al. : A new method for monitoring abnormal muscle response in hemifacial spasm: a prospective study. Clin Neurophysiol 129 : 1490-1495, 2018

22. Legatt $A D:$ Mechanisms of intraoperative brainstem auditory evoked potential changes. J Clin Neurophysiol 19 : 396-408, 2002 
23. Martin WH, Stecker MM : ASNM position statement: intraoperative monitoring of auditory evoked potentials. J Clin Monit Comput 22 : 75-85, 2008

24. Møller AR : Vascular compression of cranial nerves: II: pathophysiology. Neurol Res 21 : 439-443, 1999

25. Møller AR, Jannetta PJ : Microvascular decompression in hemifacial spasm: intraoperative electrophysiological observations. Neurosurgery $16: 612-618,1985$

26. Møller AR, Jannetta PJ : Monitoring facial EMG responses during microvascular decompression operations for hemifacial spasm. J Neurosurg 66 : 681-685, 1987

27. Mooij JJ, Mustafa MK, van Weerden TW : Hemifacial spasm: intraoperative electromyographic monitoring as a guide for microvascular decompression. Neurosurgery 49 : 1365-1370; discussion 1370-1371, 2001

28. Nielsen VK : Pathophysiology of hemifacial spasm: I. Ephaptic transmission and ectopic excitation. Neurology 34 : 418-426, 1984

29. Oge $A E$, Yayla V, Demir GA, Eraksoy $M$ : Excitability of facial nucleus and related brain-stem reflexes in hemifacial spasm, post-facial palsy synkinesis and facial myokymia. Clin Neurophysiol 116 : 1542-1554, 2005

30. Park SK, Joo BE, Lee S, Lee JA, Hwang JH, Kong DS, et al. : The critical warning sign of real-time brainstem auditory evoked potentials during microvascular decompression for hemifacial spasm. Clin Neurophysiol 129 : 1097-1102, 2018

31. Polo G, Fischer C, Sindou MP, Marneffe V : Brain auditory evoked potentialmonitoring during microvascular decompression for hemifacial spasm: intra-operative brainstem auditory evoked potential changes and warning values to prevent hearing loss-prospective study in a consecutive series of 84 patients. Neurosurgery $54: 97-106,2004$

32. Prell J, Rampp S, Rachinger J, Scheller C, Naraghi R, Strauss C : Spontaneous electromyographic activity during microvascular decompression in trigeminal neuralgia. J Clin Neurophysiol 25 : 225-232, 2008

33. Romstöck J, Strauss C, Fahlbusch R : Continuous electromyography monitoring of motor cranial nerves during cerebellopontine angle surgery. J Neurosurg $93:$ 586-593, 2000

34. Samii M, Günther T, laconetta G, Muehling M, Vorkapic P, Samii A : Microvascular decompression to treat hemifacial spasm: long-term results for a consecutive series of 143 patients. Neurosurgery 50 : 712-718; discussion 718-719, 2002

35. Shin JC, Chung UH, Kim YC, Park Cl : Prospective study of microvascular decompression in hemifacial spasm. Neurosurgery 40 : 730-734; discussion 734-735, 1997
36. Sindou M, Mercier $P$ : Microvascular decompression for hemifacial spasm : surgical techniques and intraoperative monitoring. Neurochirurgie 64 : 133-143, 2018

37. Sindou MP : Microvascular decompression for primary hemifacial spasm. Impor-tance of intraoperative neurophysiological monitoring. Acta Neurochir Wien 147 : 1019-1026; discussion 1026, 2005

38. Son BC, Ko HC, Choi JG : Intraoperative monitoring of Z-L response (ZLR) and abnormal muscle response (AMR) during microvascular decompression for hemifacial spasm. Interpreting the role of ZLR. Acta Neurochir (Wien) $160:$ 963-970, 2018

39. Thirumala PD, Carnovale G, Habeych ME, Crammond DJ, Balzer JR : Diagnostic accuracy of brainstem auditory evoked potentials during microvascular decompression. Neurology 83 : 1747-1752, 2014

40. Thirumala PD, Wang X, Shah A, Habeych M, Crammond D, Balzer JR, et al. : Clinical impact of residual lateral spread response after adequate microvascular decompression for hemifacial spasm: a retrospective analysis. Br J Neurosurg 15 : 818-822, 2015

41. Tobishima $\mathrm{H}$, Hatayama $\mathrm{T}$, Ohkuma $\mathrm{H}$ : Relation between the persistence of an abnormal muscle response and the long-term clinical course after microvascular decompression for hemifacial spasm. Neurol Med Chir (Tokyo) 54 : 474-482, 2014

42. von Eckardstein $K$, Harper C, Castner M, Link M : The significance of intraoperative electromyographic "lateral spread" in predicting outcome of microvascular decompression for hemifacial spasm. J Neurol Surg B Skull Base 75 : 198-203, 2014

43. Wilkinson MF, Kaufmann AM : Monitoring of facial muscle motor evoked potentials during microvascular decompression for hemifacial spasm: evidence of changes in motor neuron excitability. J Neurosurg 103 : 64-69, 2005

44. Xu XL, Zhen XK, Yuan Y, Liu HJ, Liu J, Xu J, et al. : Long-term outcome of repeat microvascular decompression for hemifacial spasm. World Neurosurg 110 : e989-e997, 2018

45. Yamashita S, Kawaguchi T, Fukuda M, Suzuki K, Watanabe M, Tanaka R, et al. : Lateral spread response elicited by double stimulation in patients with hemifacial spasm. Muscle Nerve $25: 845-849,2002$

46. Yang $M$, Zheng $X$, Ying $T$, Zhu J, Zhang W, Yang $X$, et al. : Combined intraoperative monitoring of abnormal muscle response and Z-L response for hemifacial spasm with tandem compression type. Acta Neurochir (Wien) 156 : 1161-1166; discussion 1166, 2014

47. Zheng $X$, Hong $W$, Tang $Y$, Ying $T$, Wu Z, Shang $M$, et al. : Discovery of a new waveform for intraoperative monitoring of hemifacial spasms. Acta Neurochir (Wien) 154 : 799-805, 2012 